\title{
Scalable Intersample Interpolation Architecture for High-channel-count Beamformers
}

Tomov, Borislav Gueorguiev; Nikolov, Svetoslav I; Jensen, Jørgen Arendt

Published in:

Proceedings of the 2011 IEEE International Ultrasonics Symposium

Link to article, DOI:

10.1109/ULTSYM.2011.0091

Publication date:

2011

Document Version

Early version, also known as pre-print

Link back to DTU Orbit

Citation (APA):

Tomov, B. G., Nikolov, S. I., \& Jensen, J. A. (2011). Scalable Intersample Interpolation Architecture for Highchannel-count Beamformers. In Proceedings of the 2011 IEEE International Ultrasonics Symposium (pp. 381384). IEEE. https://doi.org/10.1109/ULTSYM.2011.0091

\section{General rights}

Copyright and moral rights for the publications made accessible in the public portal are retained by the authors and/or other copyright owners and it is a condition of accessing publications that users recognise and abide by the legal requirements associated with these rights.

- Users may download and print one copy of any publication from the public portal for the purpose of private study or research.

- You may not further distribute the material or use it for any profit-making activity or commercial gain

- You may freely distribute the URL identifying the publication in the public portal 
Paper presented at the IEEE International Ultrasonics Symposium, Orlando Florida, 2011:

\section{Scalable Intersample Interpolation Architecture for High-channel- count Beamformers}

Borislav Georgiev Tomov ${ }^{1}$

Svetoslav Ivanov Nikolov ${ }^{2}$

Jorgen Arendt Jensen ${ }^{1}$

${ }^{1}$ Center for Fast Ultrasound Imaging,

Biomedical Engineering group, Department of Electrical Engineering, Bldg. 349,

Technical University of Denmark, DK-2800 Kgs. Lyngby, Denmark

${ }^{2}$ R\&D Applications \& Technologies, BK Medical, DK-2730 Herlev, Denmark

To be published in Proceedings of IEEE International Ultrasonics Symposium, Orlando Florida, 2011. 


\title{
Scalable Intersample Interpolation Architecture for High-channel-count Beamformers
}

\author{
Borislav Georgiev Tomov ${ }^{1}$, Svetoslav Ivanov Nikolov ${ }^{2}$ and Jørgen Arendt Jensen ${ }^{1}$ \\ ${ }^{1}$ Center for Fast Ultrasound Imaging, Dept. of Elec. Eng. Bldg. 349, \\ Technical University of Denmark, DK-2800 Lyngby, Denmark \\ ${ }^{2}$ R\&D Applications \& Technologies, BK Medical, DK-2730 Herlev, Denmark
}

\begin{abstract}
Modern ultrasound scanners utilize digital beamformers that operate on sampled and quantized echo signals. Timing precision is of essence for achieving good focusing. The direct way to achieve it is through the use of high sampling rates, but that is not economical, so interpolation between echo samples is used. This paper presents a beamformer architecture that combines a band-pass filter-based interpolation algorithm with the dynamic delay-and-sum focusing of a digital beamformer. The reduction in the number of multiplications relative to a linear perchannel interpolation and band-pass per-channel interpolation architecture is respectively $58 \%$ and $75 \%$ beamformer for a 256-channel beamformer using 4-tap filters. The approach allows building high channel count beamformers while maintaining high image quality due to the use of sophisticated intersample interpolation.
\end{abstract}

\section{INTRODUCTION}

Medical ultrasound imaging is a well-established diagnostic modality, characterized by short preparation time, lack of side effects, portability and a low price compared to other imaging modalities. The vast majority of commercial ultrasound scanners feature a digital beamformer and employ a delay-and-sum focusing of the digitized echo signals from the tissue [1]. The quality of the focusing is directly dependent on the timing precision with which it is done [2].

For perfect alignment of the echo signals, echo samples for specific moments in time must be available from all receive channels of a beamformer. One way to provide them (with small error) is to sample the echo signal at very high frequency, but that is not economical. Another way for achieving focusing precision is to use interpolation between the input samples. Experiments have been done with linear, polynomial and bandpass filtering interpolation [3]. Linear interpolation is often used, since it requires the least amount of hardware compared to other interpolation techniques.

A filter-based interpolation provides much better results than linear interpolation, but requires several multiplication operations per sample (several multipliers per input channel). Also, several sets of filtering coefficients must be kept by each filter, and a set is selected for use for each output sample, depending on its intersample position (the fractional part of the focusing delay).

Using large number of channels in ultrasound beamformers provides significant benefits, such as higher signal-to-noise ratio, better focusing, and the ability to handle 2-dimensional arrays that allow 3-dimensional (volume) imaging. In such

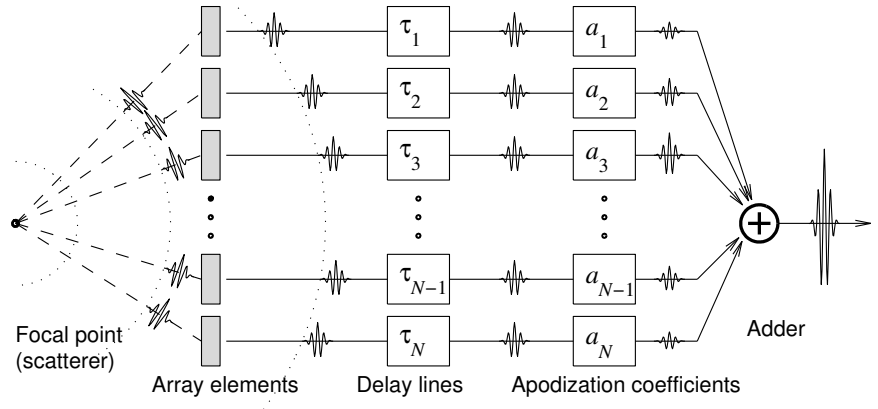

Fig. 1. Delay-and-sum focusing.

beamformers, filter-based interpolation would require linearly increasing amounts of logic and memory for its implementation.

This paper presents methods for combining the filter-based interpolation with the focusing and summing logic, thus greatly reducing the computational and the coefficient storage demands in high channel count systems. The principles and the beamformer architectures are presented in Section II, and an image quality comparison to a conventional architecture is presented in Section III.

\section{Computational ALGORithm}

\section{A. Digital beamformer operation}

A digital beamformer samples and quantizes the received echo signals using analog-to-digital converters (ADCs) and subsequently applies weights on the samples across the channels. Samples are picked from each channel according to a delay profile for the target output sample, and summed. The principle is illustrated in Fig. 1. For an $I$-channel beamformer, the output samples are summed as follows:

$$
s_{o}(n)=\sum_{i=1}^{I} s_{i}\left(i, d_{N}(i, n)\right) w(i, n)
$$

where $s_{i}$ is the sampled echo signal signal, $i$ is a channel index, $n$ is the sample index, $w$ is a weighting coefficient and $d_{N}$ is an input sample index, calculated as the rounded to an integer part of flight time converted in clock cycles of the sampling frequency $f_{s}$ :

$$
d(i, n)=\frac{f_{s}\left(p_{f}(i, n)+p_{r}(i, n)\right)}{c}
$$



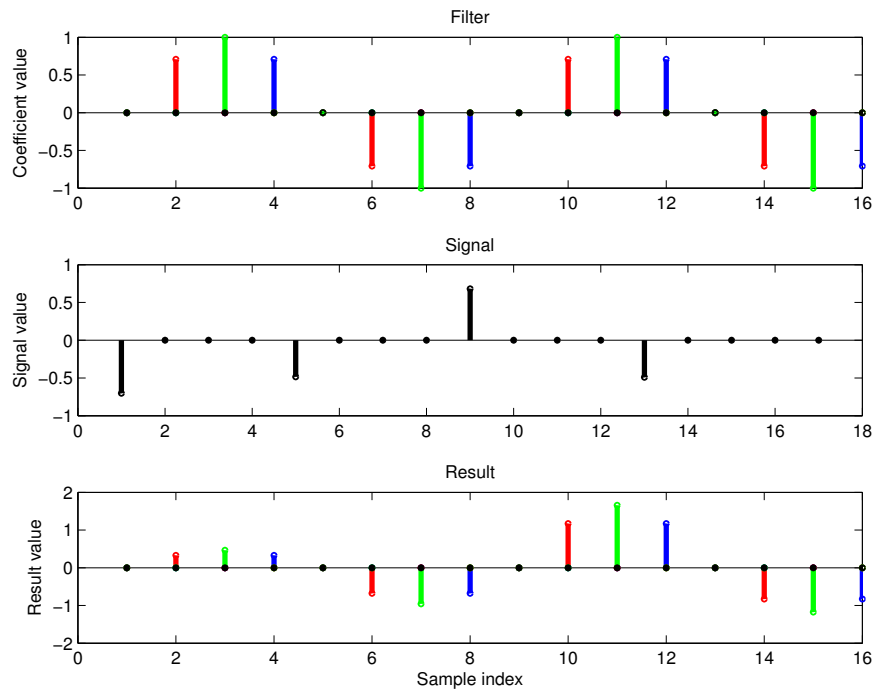

Fig. 2. Filtering of an upsampled signal: The differently colored subsets of the filter coefficients create the identically colored output samples.

where $p_{f}$ is the distance between the beam origin and the current focal point, $p_{r}$ is the distance between the focal point and the receiving element, and $c$ is the speed of sound. This method only picks out the nearest neighbor sample and leads to large sidelobes in the point spread function [3]. Some form for interpolation is, thus, needed.

\section{B. Intersample interpolation through filtration}

A signal can be upsampled by inserting zeros between consecutive input samples. Applying a bandpass filter on the result generates an upsampled output stream containing samples for time instances between those of the samples of the input signal (Fig. 2). The bandpass filter can be split into coefficient sets, which are used to selectively produce inter-sample values. Filtering with such coefficient sets is used for implementing sub-sample timing precision in a digital beamformer. Every input channel of the digital beamformer has its own filter block with selectable coefficient sets (illustrated in Fig. 3). A highchannel-count beamformer would require a large number of multipliers and RAM for storing of these identical groups of filter sets.

On each beamformer channel, a filter is applied on the input samples once per output sample. For channel $i$ out of $I$ total channels, the operation is

$$
s_{o}(i, n)=\sum_{k=1}^{K} s_{i}\left(i, j\left(k, d_{N}(i, n)\right)\right) g\left(k, M_{F}(i, n)\right)
$$

where $k$ is a filter coefficient index in a $K$-tap filter, $s_{o}$ is an output sample, $s_{i}$ is an input sample, $n$ is an output sample index, $j$ is a local sample index for the current filtration operation, $d_{N}$ is the integer part of the focusing index, and $g$ is a filter coefficient. $M_{F}$ is a filter set index, which depends on the fractional part of the $d(i, n)$, as calculated in 2 . When precision of $\frac{1}{P}$ of a sampling interval is desired, $M_{F}$ can

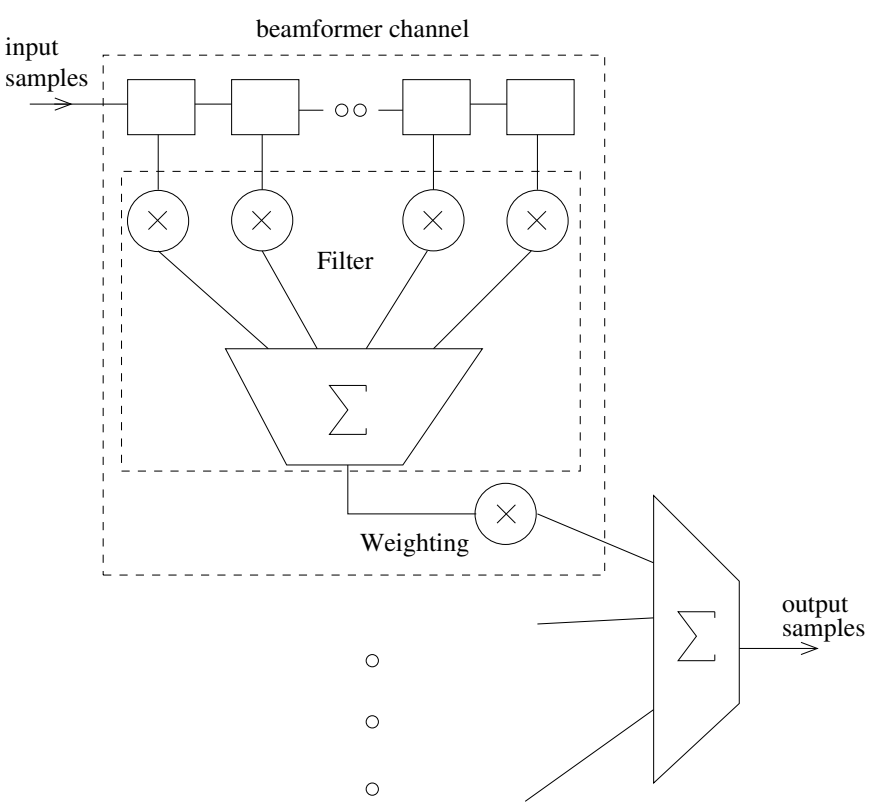

Fig. 3. FIR-filter-interpolation beamformer.

take values between 1 and $P$, thus selecting one out of the necessary $P$ interpolation filters.

A focused sample $s_{f_{o}}$ consists of the sum of apodized channel contributions:

$$
s_{f o}(n)=\sum_{i=1}^{I} s_{o}(i, n) w(i, n)
$$

where $w$ is a channel weighting coefficient, whose magnitude depends on the channel index and the depth from which the echo samples arrive.

By substituting $s_{o}$ from (3), the expression for the focused sample becomes

$s_{f o}(n)=\sum_{i=1}^{I} \sum_{k=1}^{K}\left(s_{i}\left(i, j\left(k, d_{N}(i, n)\right)\right) g\left(k, M_{F}(i, n)\right)\right) w(i, n)$

For every subset of channels $\left[i_{b}: i_{e}\right]$ for which the filter set indexes $M_{F}=M$ are the same, the expression describing the sum for that subset is

$$
\begin{aligned}
s_{f o}(n) & =\sum_{i=i_{b}}^{i_{e}} \sum_{k=1}^{K}\left(s_{i}\left(i, j\left(k, M_{F}(i, n)\right)\right) g(k, M)\right) w(i, n)= \\
& =\sum_{k=1}^{K} g(k, M) \sum_{i=i_{b}}^{i_{e}} s_{i}\left(i, j\left(k, d_{F}(i, n)\right)\right) w(i, n)
\end{aligned}
$$

The whole set of channels can be divided into the mentioned subsets, though some subsets will consist of only one channel. 


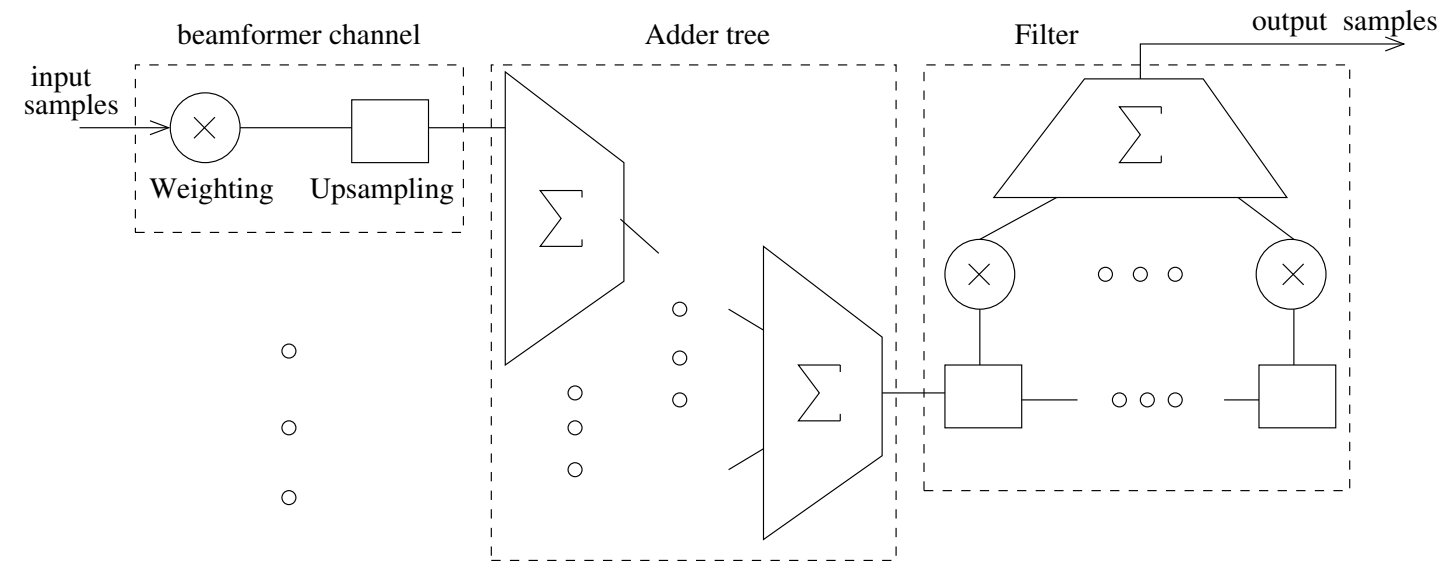

Fig. 4. Beamformer architecture utilizing non-uniform upsampling before summing across channels and filtration.

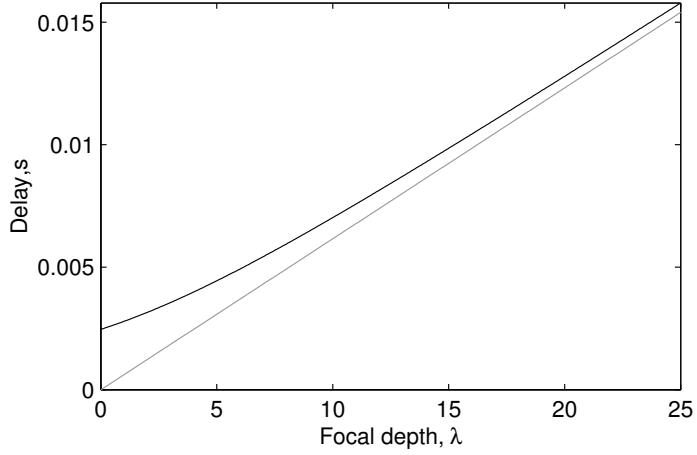

Fig. 5. Example for focusing delay development for the center element (gray) and an outer element (black) of a phased array transducer.

\section{Filtration of the sum of non-uniformly upsampled input streams}

We suggest an architecture that moves the filtration after the sum across channels, using a tree-like pipelined adder structure, operating at a higher frequency (illustrated on Fig. 4). The signal from each input channel is upsampled $P$ times, where $P$ is the number of subsample positions that provide a desired timing precision. The intersample interpolation filters, used in a per-channel filtration, here are combined back into the filter from which they are made, and that one is applied on the sum of upsampled input streams at the output sample rate. Whenever the focusing demands it, an upsampled input stream is delayed or advanced relative to the others by a sample. This introduces an error in the filtration, as the non-zero samples on the affected stream will not be equally spaced, and their filtration will not be made using filter coefficients belonging to the same interpolating filter.

A focusing index development is illustrated in Fig. 5. It shows that for the element at the image line origin, the focusing indexes grow in a straight line, meaning that the intersample distance (in time) stays the same. The filtration of that sample stream does not introduce errors. The nonuniform upsampling is required for elements for which the delay curve is not a straight line. The error has a random nature, as every imperfection may add or subtract from the
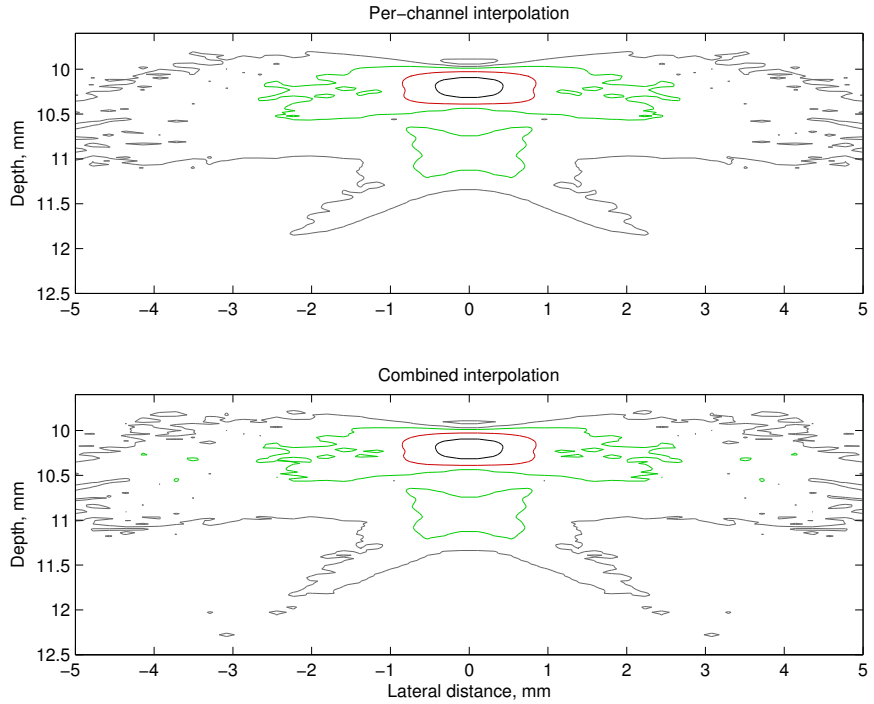

Fig. 6. Contour plot of the point spread function for depth of $1 \mathrm{~cm}$, with isolines at $-6,-20,-40$, and $-60 \mathrm{~dB}$ relative to the peak.

ideal filtration result.

\section{RESULTS}

The effect of the error, introduced by non-uniform upsampling, is investigated by simulating ultrasound imaging with Field II [4], [5] of three point reflectors, placed 1, 4, and 7 $\mathrm{cm}$ away from a 128 -element $\lambda$-spaced $7 \mathrm{MHz}$ linear array, emitting a 2-cycle pulse at the transducer center frequency. The transmit focal point is at depth of $4 \mathrm{~cm}$. Dynamic receive focusing is used with Hamming apodization and expanding aperture with an F-number of 2. An image consisting of 48 lines was created. Contour plots of the point reflector images are shown in Fig. 6 for depth of $1 \mathrm{~cm}$, in Fig. 7 for depth of $4 \mathrm{~cm}$, and in Fig. 8 for depth of $7 \mathrm{~cm}$. Black, red, green and gray isolines indicate levels of $-6,-20,-40$, and $-60 \mathrm{~dB}$ relative to the peak. Per-channel interpolation and combined interpolation are compared.

For a 256-channel beamformer using 4-tap interpolation filters and 1/16th intersample precision, the length of the 

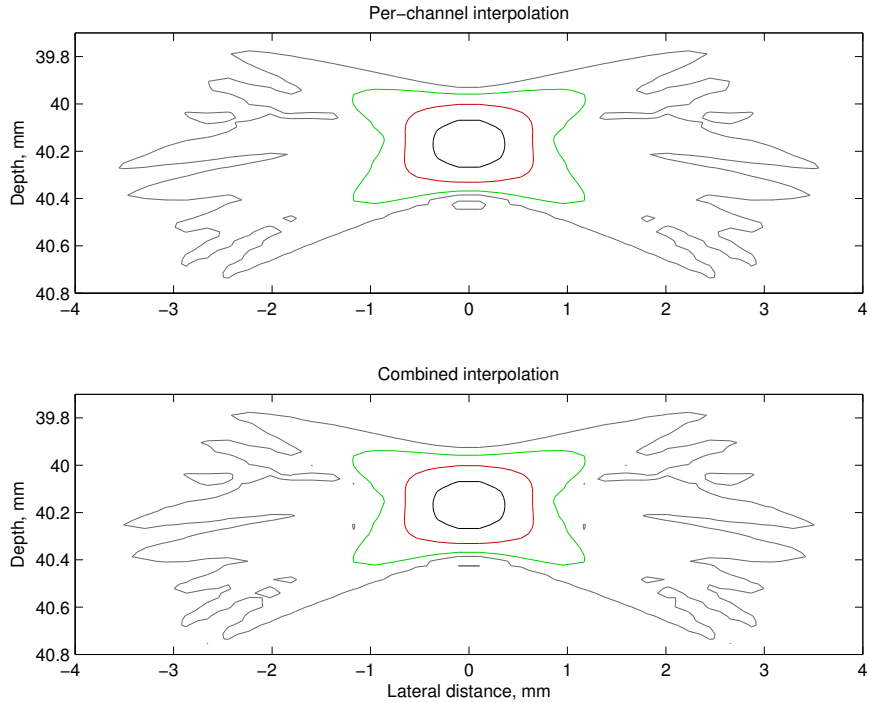

Fig. 7. Contour plot of the point spread function for depth of $4 \mathrm{~cm}$, with isolines at $-6,-20,-40$, and $-60 \mathrm{~dB}$ relative to the peak.
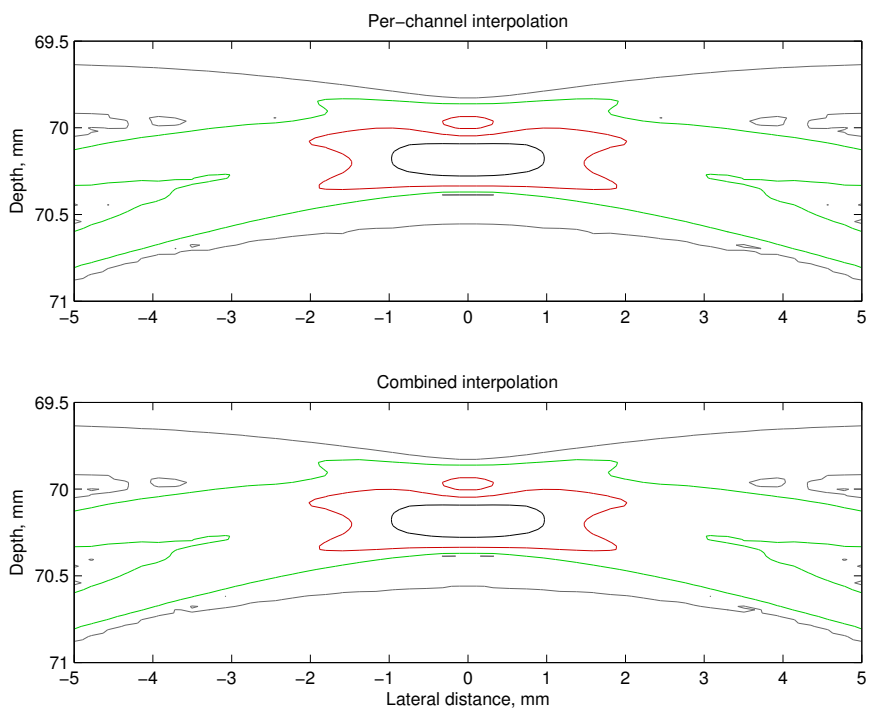

Fig. 8. Contour plot of the point spread function for depth of $7 \mathrm{~cm}$, with isolines at $-6,-20,-40$, and $-60 \mathrm{~dB}$ relative to the peak.

combined filter will be 64 coefficients (requiring 64 multipliers or multiplication operations). In all cases, a multiplier is necessary for each channel for implementing apodization (weighting). Linear interpolation requires two multiplications per channel per output sample. The combined architecture requires $256+64=320$ multiplications per output sample versus $256 *(2+1)=768$ when using per-channel linear interpolation (58.3\% savings) and $256 *(4+1)=1280$ when using per-channel bandpass interpolation (75\% savings). For a 1024channel design, the savings are correspondingly $64.6 \%$ (1088 vs. 3072$)$ and $78.7 \%$ (1088 vs. 5120).

\section{DISCUSSION}

The introduced error through non-uniform upsampling is largest in the situation where the intersample distance differs most to that for the channel at the beam origin. The error decreases as the delay profile of a channel straightens and starts approaching the straight line that is the delay profile of the center channel (illustration in Fig. 5). The use of expanding aperture and windowing means leads to avoiding the situations with worst-case injected error. The simulation shows very small deterioration in PSF, mainly in low depths.

\section{CONClusion}

The post-delay band-pass interpolation provides the precision of band-pass interpolation, combined with computational requirements that are much lower than those of linear interpolation, making it suitable for use in high-channel-count beamformers.

\section{ACKNOWLEDGMENT}

This work was supported by grant 9700883,9700563 and 26-04-0024 from the Danish Science Foundation and by B-K Medical Aps. This project is also supported by grant 0242008-3 from the Danish Advanced Technology Foundation

\section{REFERENCES}

[1] B. D. Steinberg. Digital beamforming in ultrasound. IEEE Trans. Ultrason., Ferroelec., Freq. Contr., 39:716-721, 1992.

[2] S. Holm and K. Kristoffersen. Analysis of worst-case phase quantization sidelobes in focused beamforming. IEEE Trans. Ultrason., Ferroelec., Freq. Contr., 39:593-599, 1992.

[3] J. Kortbek, H. Andresen, S. Nikolov, and J. A. Jensen. Comparing interpolation schemes in dynamic receive ultrasound beamforming. In Proc. IEEE Ultrason. Symp., pages 1972-1975, 2005.

[4] J. A. Jensen. Field: A Program for Simulating Ultrasound Systems. Med. Biol. Eng. Comp., 10th Nordic-Baltic Conference on Biomedical Imaging, Vol. 4, Supplement 1, Part 1:351-353, 1996.

[5] J. A. Jensen and N. B. Svendsen. Calculation of Pressure Fields from Arbitrarily Shaped, Apodized, and Excited Ultrasound Transducers. IEEE Trans. Ultrason., Ferroelec., Freq. Contr., 39:262-267, 1992. 